\title{
Multi-Joint Servo Systems with Backlash Compensation and Input Constraints
}

\author{
Fei $\mathrm{Xu}^{1, \mathrm{a}^{*}}$ and Liping Wang ${ }^{2, \mathrm{~b}}$ \\ ${ }^{1}$ Xijing University, Xi'an, Shaanxi, China \\ ${ }^{2}$ Xijing University, Xi'an, Shaanxi, China \\ axofun@qq.com, bwangliping@xijing.edu.cn \\ *The corresponding author
}

Keywords: Backlash nonlinearity; Convergence; Iterative learning control; Input constraints; Backstepping control

\begin{abstract}
To improve the robustness and accuracy of the traditional backstepping control, a iterative learning control is proposed and the PMSM servo actuator system is modeled. The dead-zone model is approximated by a continuously differentiable functions, in which the fitting errors and model uncertainties are regarded as disturbance-like terms. Therefore, the continuous nonlinear model with uncertain perturbation can achieve accurate compensation of backlash nonlinearity. The finite-time convergence condition of the closed-loop system has been proved by the Lapunov theory. The result shows that the tracking error converge to an arbitrarily small residual within finite time and achieve the precise compensation of the backlash.
\end{abstract}

\section{Introduction}

Backlash nonlinearity, caused by the gap between moving parts of the mechanical transmission mechanism, influences the system dynamic and steady-state precision for the power transmission process. For it is nondifferentiable and hard to accurately measure, to achieve control compensation is much difficult. Since the 1940s, the study of backlash nonlinearity is along with the development of control theory[1][2]. With the rapid expanding of application of high precision servo system and nonlinear control theory, the increasing maturity of backlash control system has attracted a lot of well-known scholars, whose research has enhanced the control quality.However, for the widely used electromechanical actuating system, and the inverse model compensation method can not be applied to the control input of the control system.

It is more realistic to describe the torque transfer relationship with the dead zone of the backlash model[3], so the non-linear system with backlash can be regarded as a non-smooth Sandwich System[4]. However, the model structure is so complex that it makes the control become difficult.A fuzzy function[5] is used to approximate the unknown nonlinear function in the backstepping process to simplify the controller design. In this paper, the iterative learning control method of backlash nonlinear compensation with control input is designed by using the repetitive characteristics of motor servo mechanism, whose convergence of iterative learning control law is strictly mathematically proved. The simulation results validate the effectiveness of the proposed method.

\section{Backlash Nonlinear Electric Servo Mechanism Model}

Electric servo mechanism is composed of drive and load part, the state equation of drive part is:

$$
\frac{d \omega}{d t}=\frac{T_{\mathrm{e}}}{J}-\frac{b_{\mathrm{m}}}{J} \omega-\frac{T_{1}}{J}
$$

Where $\theta$ is the rotor mechanical angular displacement, $\omega$ is the rotor mechanical angular velocity, $T_{\mathrm{e}}$ is output torque, $J$ is the converted equivalent moment of inertia of the motor shaft, $b_{\mathrm{m}}$ is the viscous friction coefficient, and $T_{1}$ is the converted load torque of the motor shaft. 
The equation of load dynamic part is:

$$
J_{1} \ddot{\theta}_{1}+b_{1} \dot{\theta}_{1}=\eta T_{1}+T_{\mathrm{f}}
$$

Where $J_{1}$ is load moment of inertia, $\theta_{1}$ is load output Angle, $b_{1}$ is load viscous damping coefficient, $\eta$ is transmission reduction ratio, $T_{\mathrm{f}}$ is disturbance torque. Because of the existence of backlash nonlinearity, the relationship between the load torque $T_{1}$ and the relative displacement actuator can be represented by the dead-zone model:

$$
T_{1}=\left\{\begin{array}{cc}
k(\Delta \theta-\alpha) & \Delta \theta>\alpha \\
0 & |\Delta \theta| \leq \alpha \\
k(\Delta \theta+\alpha) & \Delta \theta<-\alpha
\end{array}\right.
$$

Where, $\Delta \theta=\theta-\eta \theta_{1}, 2 \alpha$ is the backlash width, $k$ is primarily driven gear meshing stiffness coefficient. Equation (3) can be simplified as $T_{1}=k \delta$, where $\delta$ is the dead zone function. Due to the exist of the backlash, the system discontinuity makes it difficult to design the controller, therefore, a continuously differentiable functions fitting dead zones is defined:

$$
T_{\mathrm{s}}=k \Delta \theta+4 k \alpha \frac{e^{-\gamma \Delta \theta}-1}{e^{-\gamma \Delta \theta}+1}
$$

Where $\gamma$ is a constant. Define the fitting error is:

$$
T_{\Delta}=T_{1}-T_{\mathrm{s}}=T_{1}-k \Delta \theta-4 k \alpha \varepsilon(\Delta \theta)
$$

where, $\varepsilon(\Delta \theta)=\left(e^{-\gamma \Delta \theta}-1\right) /\left(e^{-\gamma \Delta \theta}+1\right)$. From (5) we obtain:

$$
T_{1}=k\left(\theta-\eta \theta_{1}\right)+4 k \alpha \varepsilon\left(\theta-\eta \theta_{1}\right)+T_{\Delta}
$$

Defining load angular velocity as $\omega_{1}=\dot{\theta}_{1}$, and substituting Equations (2) and (6) into whose derivative, then we have:

$$
\begin{aligned}
& \frac{d \omega_{1}}{d t}=-\frac{\eta^{2} k}{J_{1}} \theta_{1}-\frac{b_{1}}{J_{1}} \omega_{1}+\frac{\eta k}{J_{1}} \theta+\frac{4 k \alpha \eta \varepsilon(\Delta \theta)}{J_{1}}+d_{1} \\
& d_{1}=\frac{\eta}{J_{1}} T_{\Delta}+\frac{T_{\mathrm{f}}}{J_{1}}
\end{aligned}
$$

Substituting Equation (6) into Equation (1), then the equations of driving part can be rewritten as:

$$
\begin{aligned}
& \frac{d \omega}{d t}=\frac{\eta k}{J} \theta_{1}-\frac{k}{J} \theta-\frac{b_{\mathrm{m}}}{J} \omega+T_{\mathrm{e}}-\frac{4 k \alpha \varepsilon(\Delta \theta)}{J}+d_{2} \\
& d_{2}=-\frac{T_{\Delta}}{J}
\end{aligned}
$$

Defining state variables as $\boldsymbol{x}_{i}=\left[\theta_{1}, \omega_{1}, \theta, \omega\right]$, so the Equations (1-2) and (7-8) describle servo state equations of nonlinear backlash as follows:

$$
\begin{aligned}
& \frac{d \omega_{1}}{d t}=-\frac{\eta^{2} k}{J_{1}} \theta_{1}-\frac{b_{1}}{J_{1}} \omega_{1}+\frac{\eta k}{J_{1}} \theta+\frac{4 k \alpha \eta \varepsilon(\Delta \theta)}{J_{1}}+d_{1} \\
& \frac{d \omega}{d t}=\frac{\eta k}{J} \theta_{1}-\frac{k}{J} \theta-\frac{b_{\mathrm{m}}}{J} \omega-\frac{4 k \alpha \varepsilon(\Delta \theta)}{J}+\frac{T_{\mathrm{e}}}{J}+d_{2}
\end{aligned}
$$

According to Equations (7) and (8), when fitting dead zone features with differentiable function, the fitting error $T_{\Delta}$ and interference $T_{\mathrm{f}}$ can be merged into $d_{1}$, which, along with the original system interference item, is uncertain form, or similar interference item. 


\section{Controller Design}

Multi-joint Servo System Model. Equation (9) of backlash model of mechanical and electrical servo mechanism is extended to a MIMO system. Considering that the multi-joint servo system is driven by $n$ motors, the position variables can be described as $\boldsymbol{\theta}=\left[\theta_{1}, \ldots, \theta_{n}\right]^{T}$, the $n$th passive part position variable is $\boldsymbol{\theta}_{l}=\left[\theta_{11}, \ldots, \theta_{1 n}\right]^{T}$, the subscript $i=(1,2, \ldots n)$ means the $i$ th joint corresponding servo. Then the multi-joint servo system equation can be described as:

$$
\begin{aligned}
& D_{m} \ddot{\theta}+b_{m} \dot{\theta}=T_{e}-T_{l} \\
& D_{l} \ddot{\theta}_{l}+b_{l} \dot{\theta}_{l}=\eta T_{l}+T_{f} \\
& T_{l}=k \delta
\end{aligned}
$$

Equation (10) can be understood as Equation (9) on the dimension extension:

$$
\left\{\begin{array}{l}
\dot{\boldsymbol{x}}(t)=\boldsymbol{F}(\boldsymbol{x}(t))+\boldsymbol{B u}(t)+\boldsymbol{d}(t) \\
\boldsymbol{y}(t)=\boldsymbol{C} \boldsymbol{x}(t)
\end{array}\right.
$$

Where $\boldsymbol{x}(t)=\left[\boldsymbol{\theta}_{\boldsymbol{l}}, \boldsymbol{\omega}_{l}, \boldsymbol{\theta}, \boldsymbol{\omega}\right]$ is the state vector equation, $\boldsymbol{u}(t)=\boldsymbol{T}_{\boldsymbol{e}}(t)$ is vector control, $\boldsymbol{y}(t)$ is output

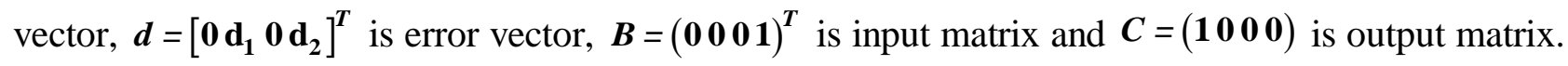

Considering a actual controller under input constrained conditions, the theory output and the actual input of the object are not consistent. For this reson, we define a saturation function $\boldsymbol{u}(t)$ :

$$
\operatorname{sat}[\boldsymbol{u}(t)]=\left\{\begin{array}{l}
-\boldsymbol{u}_{\max }(t), \boldsymbol{u}(t) \leq-\boldsymbol{u}_{\max }(t) \\
\boldsymbol{u}(t),|\boldsymbol{u}(t)|<\boldsymbol{u}_{\max }(t) \\
\boldsymbol{u}_{\max }(t), \boldsymbol{u}(t) \geq \boldsymbol{u}_{\max }(t)
\end{array}\right.
$$

Then when the input is limited, the state Equation (11) has the following form:

$$
\left\{\begin{array}{l}
\dot{\boldsymbol{x}}(t)=\boldsymbol{F}(\boldsymbol{x}(t))+\boldsymbol{B} s a t[\boldsymbol{u}(t)]+\boldsymbol{d}(t) \\
\boldsymbol{y}(t)=\boldsymbol{C} \boldsymbol{x}(t)
\end{array}\right.
$$

Control Objectives. Equation (10) described a repeated movement of multi-joint servo system. The iterative learning controller is designed under the condition of limited control input. The desired angle signal is well tracked, achieving backlash nonlinear optimal compensation.

Define function $f:[0, \mathrm{~T}] \rightarrow \mathrm{Rn}$, whose $\lambda$ - norm is

$$
\|f\|_{\lambda}=\sup _{0 \leq t \leq T}\left\{\left\|f(t) e^{-\lambda t}\right\|\right\}
$$

Where $\lambda>0 .\|\|$ is infinite norm.

\section{Simulation Results and Analysis}

The simulation is carried out in MATLAB to verify the effectiveness of the algorithm. Let's set the expected rudder angle instruction as $\varphi=30 \sin \pi t$, set rudder angle constraint range as $z_{1} \in(-1,1)$, set the controller parameters as $\kappa_{1}=\kappa_{2}=200, \kappa_{3}=\kappa_{4}=80, \lambda=20, \rho_{1}=\rho_{2}=0.1$, set the adaptive law parameters as $\gamma_{1}=\gamma_{2}=0.1$, set the initial value of the unknown parameters $F_{1} 、 F_{2}$ as 0 . Taking the conventional sliding-mode control method with quadratic Lyapunov function as reference, $V_{1}=\left(z_{0}^{2}+z_{1}^{2}\right) / 2$ is designed in the conventional algorithm, and the stability of the conclusions and control parameters remain unchanged. Then the simulation results are shown in Figure 1: 


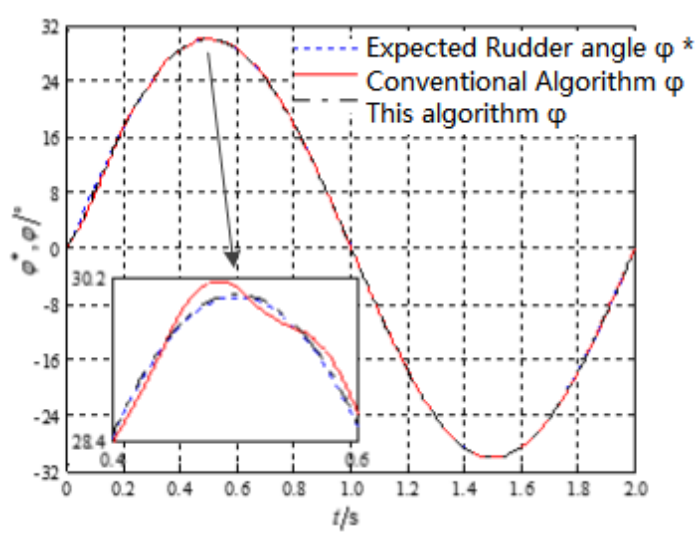

(1) Rudder angle tracking curve

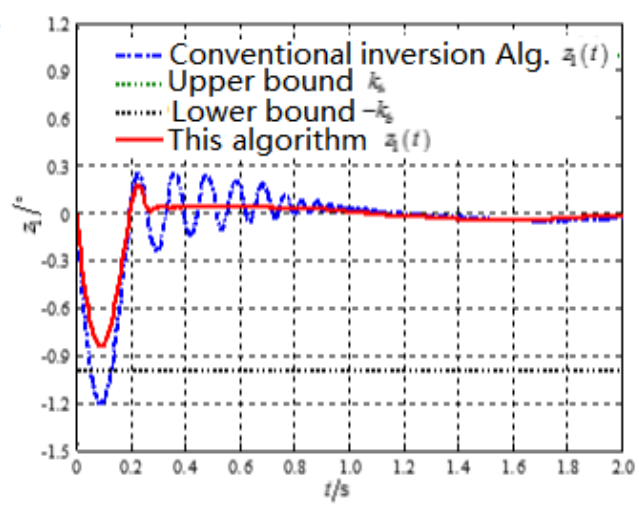

(2) $z_{1}(t)$ curves

Figure 1. Simulation results

It can be seen that this algorithm has higher control precision, no large overshoot, $z_{1}(t)$ is constrained within the setting range, the convergence is much quicker. By contrast, the conventional sliding-mode control can ensure the stability of the system as well, but can not meet the error constraints.

\section{Conclusion}

This paper proposes a control input constraints of backlash nonlinearity compensation iterative learning control method. Based on the nonlinear model of single servo, the research method is extended to the multi-joint servo system. The dead-zone model is approximated by continuously differentiable functions, and the fitting errors and model uncertainties are treated as disturbance-like terms. Therefore, the continuous nonlinear model with uncertain perturbation can realize the accurate compensation of backlash nonlinearity. Compared with the conventional backstepping control, the experimental results show that the control precision and robustness are significantly improved. Theoretical and experimental simulation results have verified the validity and rationality of this method.

\section{References}

[1] Gang T, Kokotovic P V. Adaptive control of systems with backlash[J]. Automatics, 1993, (2): 323-335.

[2] Mattias N, Per G. Controlling mechanical systems with backlash; a survey[J]. Automatics, 2002, (10): 633-1649.

[3] Zhou J, Wen C Y, Zhang Y. Adaptive backstepping control of a class of uncertain nonlinear systems with unknown backlash-like hysteresis[J]. IEEE Transactions on Automatic Control, 2004, (10): 1751-1759.

[4] Zhou J, Zhang C J, Wen C Y. Robust adaptive output control of uncertain onlinear plants with unknown backlash nonlinearity [J]. IEEE Transactions on Automatic Control, 2007, (3): 503-509.

[5] Liu Y J, Tong S C. Adaptive fuzzy control for a class of nonlinear discrete-time systems with backlash[J]. IEEE Transactions on Fuzzy Systems, 2014, (s): 1359-136s.

[6] Wang H Q, Chen B, Liu K F, et al. Adaptive neural tracking control for a class of nonstrict-feedback tochastic nonlinear systems with unknown backlash-like hysteresis [J]. IEEE Transactions on Neural Networks and Learning Systems, 2014,s(s): 947-9s8. 
[7] Tarbouriech S, Queinnec I, Prieur C. Stability analysis and stabilization of systems with input backlash [J]. IEEE Transactions on Automatic Control, 2014, (2): 488-494.

[8] Philipp R, Thomas B, Miroslav B, et al. Hybrid aproach to modeling, control and state estimation of mechanical systems with backlash [J].International Journal of Control, 2007, (11): 1729-1740.

[9] Corradini M L, Orlando G. Robust stabilization of nonlinear uncertain plants with backlash or dead zone in the actuator [J]. IEEE Transactions on Control Systems Technology, 2002, (1): $1 \mathrm{~s} 8-166$.

[10]Zhu Sheng, Sun Mingxuan, He Xiongxiong. Iterative learning control of a class of nonlinear systems with input backlash[J].Acta Automatica Sinica, 2011, (8): 1014-1017. 